\title{
Over restrictive elimination of foods in children with food allergy
}

\author{
Elif Özdoğan ${ }^{1, \oplus}$, Hacer Efnan Melek ${ }^{2, \oplus}$, Betül Büyüktiryaki ${ }^{3 \oplus}$, \\ Hikmet Tekin Nacaroğlu ${ }^{4, a}$, Raziye Dut ${ }^{5 \oplus}$, Özge Soyer ${ }^{6 \oplus}$, Ümit M. Şahiner ${ }^{6 \oplus}$, \\ Ebru Arık Yılmaz ${ }^{7, b \oplus}$, A. Deniz Akkaya ${ }^{8 \oplus,}$ Nuray U. Kızılkan", Cansın Saçkesen ${ }^{3 \oplus}$
}

${ }^{1}$ Koç University Faculty of Medicine, İstanbul; ${ }^{2}$ Department of Pediatrics, VM Medical Park Hospital, Kocaeli; Divisions of ${ }^{3}$ Pediatric Allergy and ${ }^{9}$ Pediatric Gastroenterology Koc University School of Medicine, Istanbul; ${ }^{4}$ Division of Pediatric Allergy and Immunology, İstanbul Medipol University, İstanbul; ${ }^{5}$ Department of Pediatrics, İstanbul Research and Training Hospital, İstanbul; ${ }^{6}$ Division of Pediatric Allergy, Hacettepe University Faculty of Medicine, Ankara; ' Division of Pediatric Allergy, Pamukkale University Faculty of Medicine, Denizli; ${ }^{8}$ Department of Dermatology, Liv Hospital, İstanbul, Turkey.

\begin{abstract}
Background. Previous studies demonstrated critical deficits in diagnosis and management of childhood food allergy (FA), and recent developments in FA research support adopting a proactive approach in FA management. Our objective was to describe FA knowledge and management patterns of pediatricians.

Method. We applied a 24-item survey to 170 general pediatricians, pediatric allergists and pediatric gastroenterologists practicing in Turkey.

Results. Some IgE-mediated symptoms of FA such as cough, urticaria, wheezing and anaphylaxis were falsely recognized as symptoms of non-IgE-mediated FA by 30\%, 29\%, 25\% and 19\% of the participants, respectively. By contrast, $50 \%$ of the participants falsely recognized bloody stool, a finding of IgE-mediated FA. Most frequently and least frequently used diagnostic tools were specific $\operatorname{IgE}(30.5 \%)$ and oral food challenge test $(1.7 \%)$, respectively. Maternal diet restrictions and infant diet restrictions were advised by $82 \%$ and $82 \%$, respectively. Percentages of physicians eliminating only 1 food were 21\%, 19\%; 2 foods were $15 \%, 11 \% ; 3$ foods were $7 \%, 8 \%$; $4-5$ foods were $8 \%, 11 \%$; 5 to 10 foods were $21 \%, 26 \%$; and $>10$ foods were $28 \%, 25 \%$ from the maternal and infant diet, respectively. Cow's milk, cheese, butter, yoghurt, baked milk products and hen's egg were the most commonly restricted items.

Conclusion. Overall, FA knowledge of pediatricians was fair. Pediatricians utilize an overly restrictive approach when advising diet eliminations in FA. Recent developments favor a more proactive approach to induce immune tolerance and need to be encouraged in pediatric clinical practice. Future educational efforts should focus on emphasizing the deleterious effects of injudicious and extensive eliminations.
\end{abstract}

Key words: diet elimination, food allergy, knowledge, survey, nutrition.

$\triangle$ Cansın Saçkesen

csackesen@ku.edu.tr • csackesen@yahoo.com

Received 9th April 2020, revised 18th May 2020, accepted 8th June 2020

* Elif Özdoğan and Hacer Efnan Melek are joint first authors.

Author institution the study took place:

a: Division of Pediatric Allergy, Bağcllar Research and Training Hospital, İstanbul.

b: Division of Pediatric Allergy, Denizli State Hospital, Denizli.
The prevalence of clinical food allergy (FA) is estimated to be $10 \%$ among preschool children globally and has been showing a steadily increasing trend..$^{1-3}$ The rise in the number of admissions of infants with FA complaints have compelled physicians to learn about and adapt to different types of FA such as IgE-mediated and non-IgE mediated FA. ${ }^{4}$ The differences between the two types include disparate ways of presentation. While some of infants with FA may present with chronic atopic dermatitis 
symptoms, others may be admitted with anaphylaxis.

The mainstay of FA treatment is avoidance of triggering foods. ${ }^{4}$ Arguably, prescribing dietary interventions, especially to children with allergic disease may often times be a challenging task. Half of the children with FA suffer from severe, acute allergic reactions and one-third have multiple food allergies. ${ }^{2}$ Therefore, it is essential to employ appropriate diagnostic tools to identify the exact offending products and educate parents and patients about strict adherence. On the other hand, the latest studies have repeatedly shown that some children with FA benefit from more liberal diets. ${ }^{5}$ In terms of prevention of FA in at-risk infants, several studies found that early introduction of food allergens significantly reduced the prevalence of FA when food allergens were included in the diet earlier on in life. ${ }^{6,7}$ Moreover, any interference with nutrition in a growing child should be well-planned due to the serious consequences of malnutrition..$^{8,9}$

In accordance with the challenges that the physicians face, being treated for FA may be a challenging period for patients and their families as well. The elimination of major foods, the risk of anaphylaxis and chronic symptoms such as eczema or failure to thrive perturb the social and psychological wellbeing of affected children. Accordingly, there is strong evidence that FA and food hypersensitivity has an impact on psychological distress and on the quality of life of children and their families. ${ }^{10,11}$

Currently only few data exist that describe practices in the diagnosis and management of FA among pediatricians in Turkey. ${ }^{12}$ Similar studies that evaluated the FA-related practices of primary care physicians (PCP) and/or pediatricians in the US, Finland, Kuwait, Australia and Croatia have consistently demonstrated significant deficits. ${ }^{13-17}$ Given the developing need for the physicians to adapt themselves to recent changes in FA management, the wide range of different clinical presentations of IgE and non-IgE mediated FA, and the impacts of FA and diet restrictions on the patient's quality of life we prepared this survey to describe the FA knowledge and practices of general pediatricians, pediatric allergists and pediatric gastroenterologists.

\section{Material and Methods}

\section{Questionnaire}

A self-administered questionnaire about childhood FA was designed and administered to general pediatricians, pediatric allergists and pediatric gastroenterologists practicing in Turkey. The 24-item questionnaire was composed of 10 questions about the demographic characteristics of the participants and 14 questions about their clinical approach to FA. Of the 14 questions that were on FA; 8 were multiple-choice, 5 were dichotomous and 1 was an open-ended question (Supplementary data).

The survey included questions about the frequency of encountering patients with FA, symptoms of IgE and non-IgE mediated FA, symptoms of FA in patients with atopic dermatitis (AD), diagnosis of $\mathrm{FA}$ in $\mathrm{AD}$, and the practices regarding dietary management of $\mathrm{FA}$.

In this study, the term FA is used to refer to both $\operatorname{IgE}$ and non-IgE FA, with relevant specifications being used if either one is referred to. The study was approved by the Koc University ethical committee on 13/07/2016 (report number: 2016.165.IRB2.092), and written informed consent was obtained from all participants.

\section{Statistical analysis}

Statistical analyses were conducted using SPSS statistics version 18 (SPSS Inc, Chicago, IL, USA). Data were manually transferred into Excel and then into SPSS version 18 for analysis. Categorical variables were presented as frequency and percentiles. Normally distributed values were expressed as means. 
Table I. Demographic characteristics of the participants.

\begin{tabular}{lc}
\hline Characteristic & Pediatricians, $\mathrm{n}(\%)$ \\
\hline Gender & \\
Female & $114(67.1)$ \\
Male & $56(32.9)$ \\
Age & \\
$25-30$ years & $54(31.8)$ \\
$30-40$ years & $64(37.9)$ \\
$40-50$ years & $38(22.5)$ \\
$>50$ years & $13(7.7)$ \\
Specialty & \\
General pediatrics & $117(69)$ \\
Pediatric allergy & $29(17)$ \\
Pediatric gastroenterology & \\
Specialty education center & $24(14)$ \\
University hospital & \\
Research and training hospital & $80(50)$ \\
Practicing center & $80(50)$ \\
Secondary Care Facility & \\
Tertiary Care Facility & \\
Current working status & $24(14.8)$ \\
Fellow in training & $138(85.2)$ \\
Specialist & \\
Faculty member & $63(38.4)$ \\
\hline
\end{tabular}

a Specialist or fellow in training

${ }^{\mathrm{b}}$ Government hospital, Private hospital

c University hospital, Research and Training Hospital

\section{Results}

A total of 170 physicians completed the survey. The characteristics of the participants are shown in Table I. Physicians who cared for patients with FA constituted $90 \%$ of the cohort.

\section{Knowledge on symptoms of FA}

Physicians were asked about the symptoms that would prompt suspicion of FA in the context of atopic dermatitis (AD) specifically, since workup in $\mathrm{AD}$ involves screening for general FA symptoms. Blood in stool (91\%), urticaria (84\%), appearance of $\mathrm{AD}$ lesions (81\%), abdominal symptoms $(72 \%)$, perioral erythema $(68 \%)$, diarrhea $(68 \%)$ and vomiting $(63 \%)$ were among the most common symptoms that made the physicians suspect FA in an infant diagnosed with AD. Other commonly identified symptoms of FA are outlined in Figure 1A. Competence of the physicians with regards to differentiating IgE-mediated and non-IgE mediated symptoms of FA are shown in Figure 1B.

\section{Diagnostic approach to $F A$}

In the diagnosis of FA, the following approaches were utilized in order of most-toleast commonly preferred: specific $\operatorname{IgE}(\mathrm{sIgE})$ $(30.5 \%)$, clinical findings $(25.8 \%)$, skin prick tests $(25.3 \%)$, response to diagnostic elimination diets $(5.3 \%)$, atopy patch testing (5\%), oral food challenge test (OFC) $(1.7 \%)$. The participants were allowed to select more than one answer. 
A

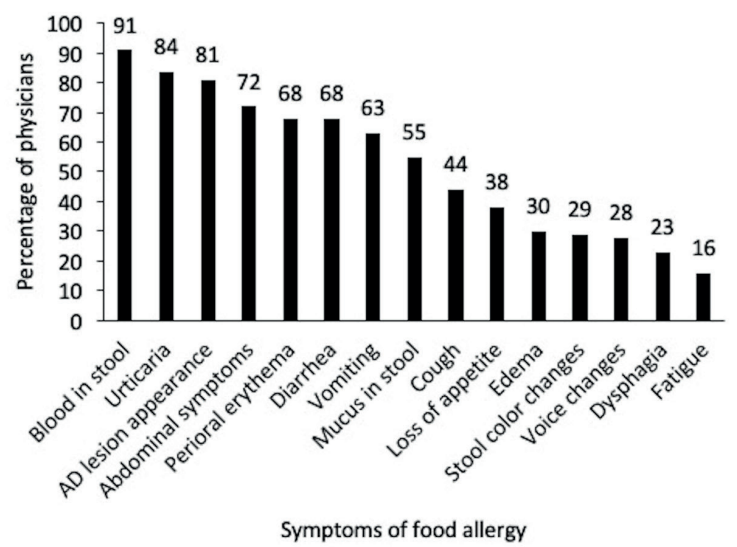

B

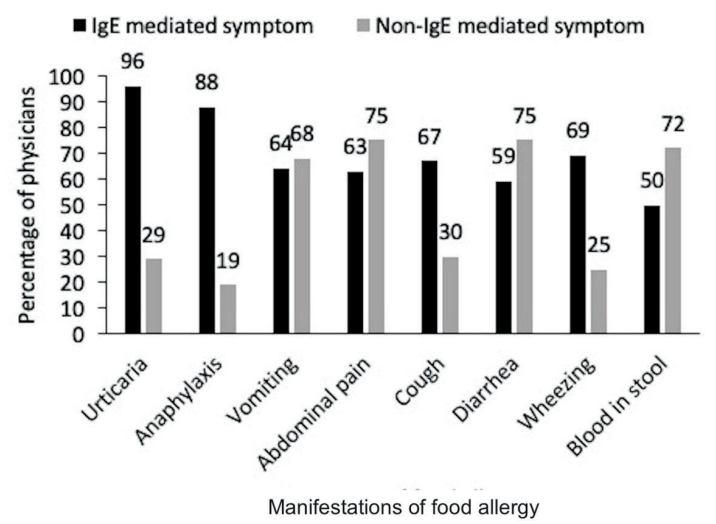

Fig. 1. Symptoms and manifestations of food allergy according to the physicians. A. Symptoms suggestive of food allergy in infants diagnosed with atopic dermatitis. B. Manifestations of IgE- and non-IgEmediated food allergy.

\section{Recommendation of maternal diet restriction (MDR) in FA}

Eighty-two percent of the physicians responded that they advise MDRs in the management of infants diagnosed with FA. Regarding the improvement of FA symptoms following a period of MDR, $72 \%, 19 \%$ and $5 \%$ selected complete clinical improvement, partial clinical improvement and no clinical improvement, respectively. (Fig. 2).

\section{Characteristics of MDRs advised in FA}

Majority of the physicians routinely restricted greater than five food products from the

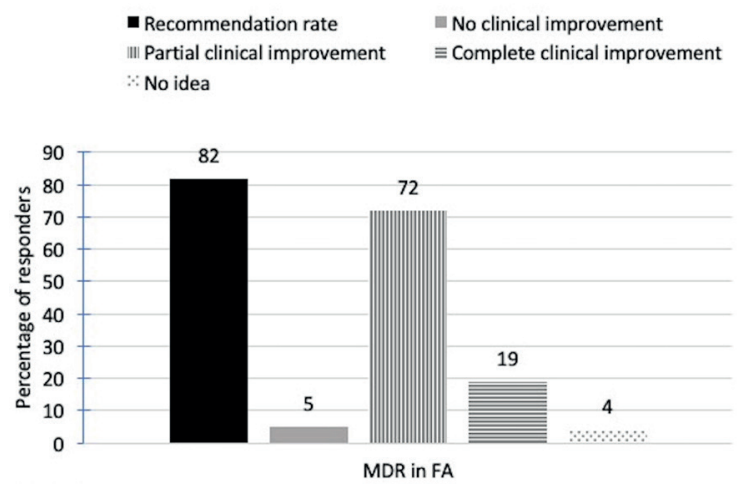

Fig. 2. Dietary management practices and perceived outcomes; MDR: maternal diet restriction, FA: food allergy.
A Maternal Diet Restriction

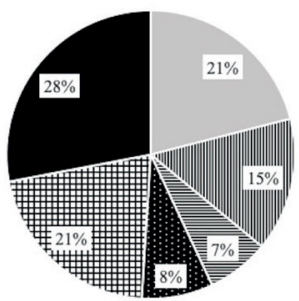

B Infant Diet Restriction

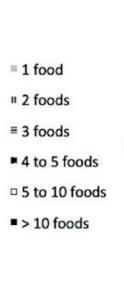

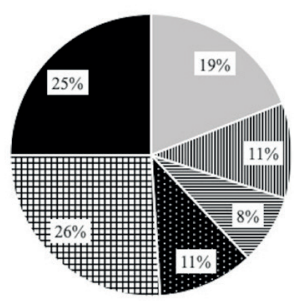

Fig. 3. Percentage of physicians eliminating different numbers of food products. A. from maternal diet in the management of FA, B. from infant's diet in the management of FA.

maternal diet: $21 \%$ restrict 5 to 10 foods, $28 \%$ restrict $>10$ foods (Fig. 3A). Cow's milk (86\%), cheese $(70 \%)$, yoghurt $(56 \%)$, butter $(56 \%)$, baked milk products (51\%), hen's egg white $(50 \%)$, goat's milk (46\%) and peanut $(43 \%)$ were the most commonly restricted products. Relatively uncommon allergens in infancy such as strawberry $(21 \%)$, tomato $(18 \%)$, cacao $(14 \%)$, orange $(12 \%)$ and blackberry $(9 \%)$ were restricted more commonly than wheat $(7 \%)$ and lentils (5\%) (Fig. 4).

Calcium supplementation was offered by $83 \%$ of the physicians to the breastfeeding mothers who were on a cow's milk $(\mathrm{CM})$ restricted diet.

\section{Recommendation of infant diet restriction (IDR) in FA}

Eighty-two percent of physicians responded that they use infant diet restriction in the 


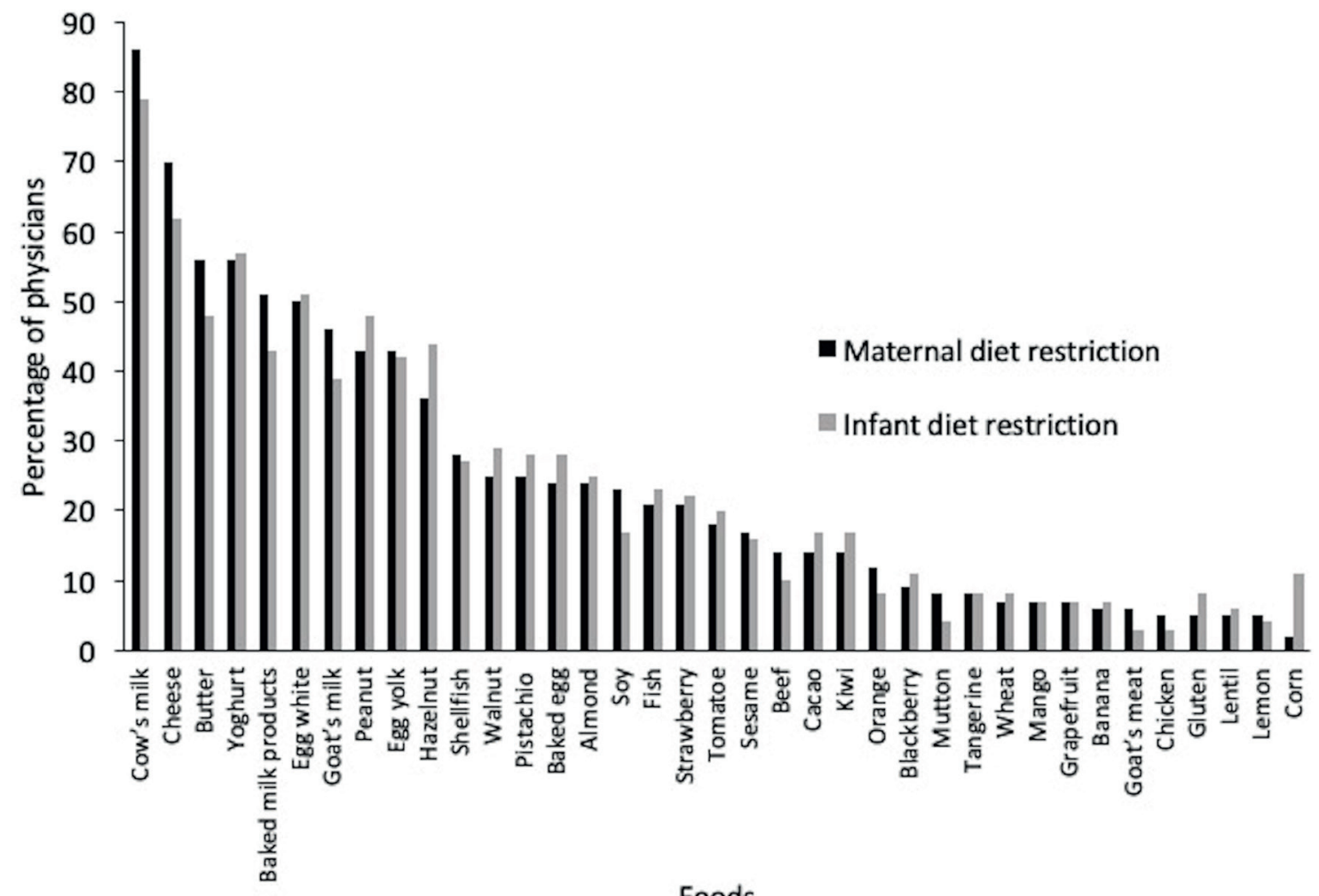

Foods

Fig. 4. Percentage of physicians eliminating different food products from the maternal and infant's diet in the management of food allergy.

management of FA. It should be noted that the IDRs employed in the management of breastfed infants with FA were noted to be empirical and not directed by allergen identification, since only a negligible percentage of the physicians declared that they use diagnostic elimination and oral food challenge (Diagnostic approach to FA).

Half of the physicians restricted 5-10 foods (26\%), or $>10$ foods (25\%) from the infant's diet (Fig. 3B). Among the blanket restrictions employed by the physicians, the most commonly restricted categories of foods were dairy products (cow's milk $79 \%$, cheese $62 \%$, yoghurt $57 \%$, butter $48 \%$, baked milk products $43 \%$ and goat's milk $39 \%$ ), peanut (48\%), tree nuts (hazelnut $44 \%$, walnut $29 \%$, pistachio $28 \%$ and almond $25 \%$ ), egg (hen's egg white 51\%, hen's egg yolk $42 \%$ and baked egg products $28 \%$ ), seafood (fish $28 \%$ and shellfish $27 \%$ ) and other foods (strawberry
$22 \%$, tomato $20 \%$, soy $17 \%$, cacao $17 \%$, kiwi $17 \%$ and sesame $16 \%$ ). (Figs 4, 5). In addition, 23\% of physicians added probiotics to the infant's diet.

\section{Introduction of complementary foods}

Physicians were asked the following two-part question regarding the time for introduction of complementary foods to the diets of infants with FA:

- Do you delay introduction of complementary foods for your patient? 23\% reported that they delay the introduction of complementary foods.

- When do you recommend the introduction of complementary foods to your patients' diet? 94\% reported that they introduce complementary foods by 6 months of age. $5 \%$ of physicians reported that they wait until 1 year of age to introduce complementary foods. 


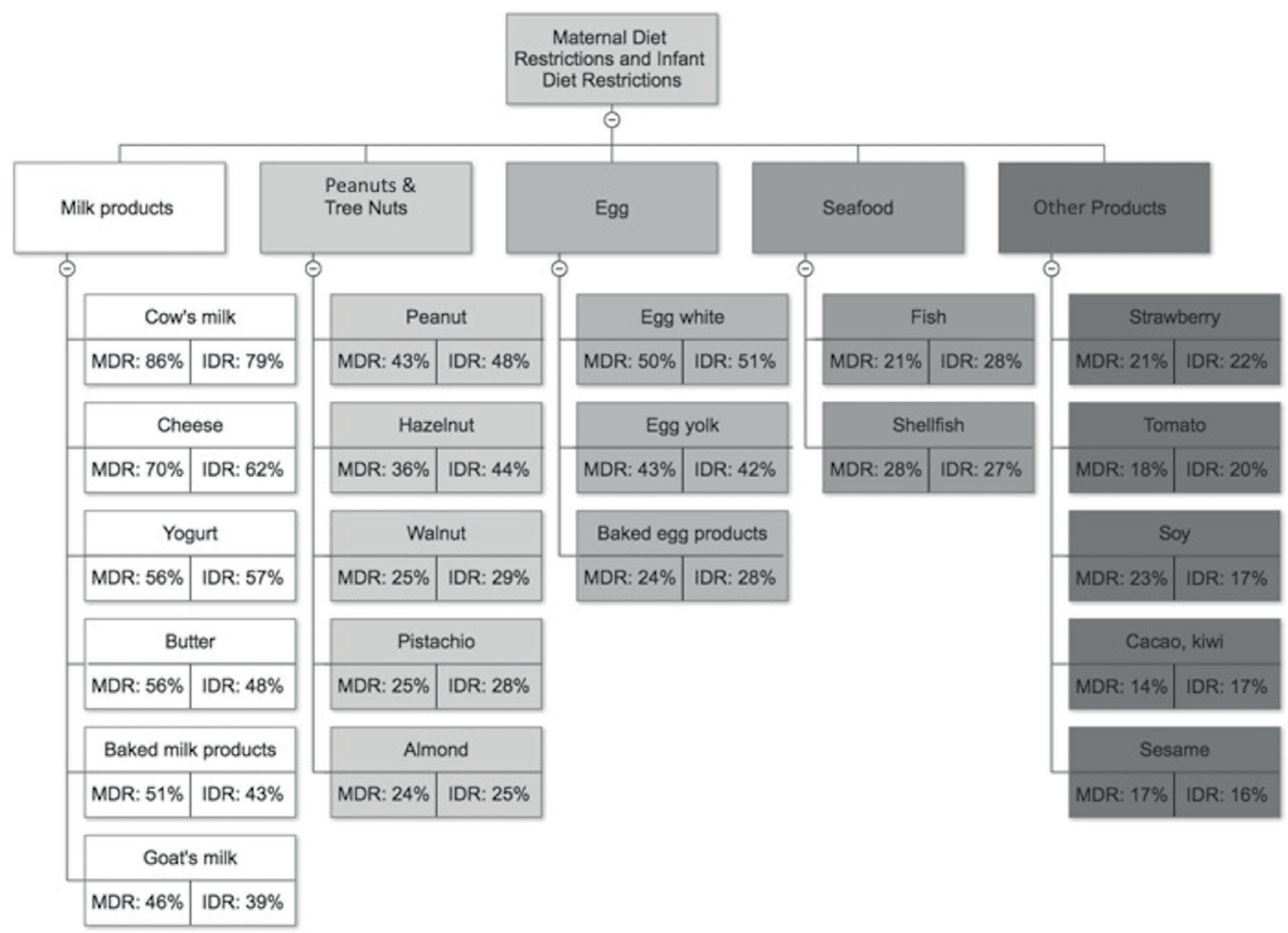

Fig. 5. Percentage of physicians restricting different food products from the maternal diet and infant's diet based on food categories.

The top 3 most common foods for introduction were vegetables (55\%), fruits (26\%) and yoghurt $(12 \%)$ in FA, respectively. Both questions in essence inquired the timing of introducing complementary foods. The contradictory results of the two questions probably means that some of the physicians introduce a small number of complementary foods.

\section{Discussion}

This study provides insights into the role and extent of nutritional management in childhood FA. The paramount finding is that pediatricians advise highly extensive and strict diet eliminations both to nursing mothers and to the infants with food allergy. This practice is incongruent with the latest clinical studies that have consistently demonstrated the benefits of more proactive therapeutic strategies. Recently, early introduction of allergenic foods to infants was shown to be effective in preventing development of FA. 6,7 Furthermore, proactive therapeutic interventions for the treatment of FA such as introduction of baked milk and baked egg products, preference of extensively hydrolyzed formula with (or without) probiotics, and use of oral immunotherapy have gained important roles. ${ }^{18-20}$ This study describes the fearful and reserved approach of the physicians to FA.

Participants' knowledge on symptoms and signs of FA was fair. However, the distinction between manifestations of IgE and non-IgEmediated FA was somehow unclear. A nonnegligible fraction falsely responded that nonIgE-mediated FA causes urticaria (30\%) and anaphylaxis $(20 \%)$. Additionally, the acute respiratory symptoms of FA were under- 
recognized by many. Such knowledge gaps in the distinction between acute reactions from chronic conditions could hinder parental education and delay the administration of lifesaving treatments, which was also noted as a significant problem among Finnish primary care physicians in the care for FA. ${ }^{21}$

Clear shortcomings in the diagnosis of FA were appreciated. The critical findings are that only one-third of the physicians take into account the clinical manifestations of the infants with suspected FA and only 1.7\% use diagnostic diet elimination followed by OFC in the diagnosis of FA. Studies investigating the FA practices in the US, Australia, Croatia, Finland and our study share the common finding that clinical diagnosis of FA is a major weakness of the PCPs and/or pediatricians. ${ }^{13-15,17}$ Internationally, physicians have a tendency to overlook clinical findings, skip OFCs and use sIgE as their only diagnostic method. Physicians should be reminded that sensitization and clinical allergy are disparate entities and that all of the current guidelines are consistent in their recommendation to perform diagnostic diet eliminations and/or OFCs as confirmatory steps in FA work-up. ${ }^{22,23}$

Moving on to the diet eliminations themselves, it was found that more than half of the physicians restrict at least 5 food products in any diet restriction they recommend to the nursing mothers and to the infants with FA. Especially regarding maternal diet restrictions for FA, this is a questionable approach for several reasons. Food antigens pass into breastmilk in minimal concentrations, hence only a small percentage of infants benefit from MDRs. ${ }^{24}$ Although cases of anaphylactic reactions upon breastfeeding have been described previously, diagnostic diet eliminations followed by OFC should be advised to identify the smaller percentage of infants that are really under risk. ${ }^{23}$

By restricting high number of food products without making patient-based modifications, Turkish pediatricians are potentially paving the way for increases in allergies. Evidence suggests that early exposure to allergenic foods reduces the risk of atopic disease through promoting regulatory T-cell pathways, especially in infants with $\mathrm{AD}$ who have impaired skin barrier. ${ }^{25}$ Observational prospective studies and randomized clinical trials investigating the effects of early exposure to CM, cooked eggs and peanut found significantly lower levels of FA with early exposure. ${ }^{6,26,27}$ Studies showing correlation of egg protein concentrations in breastmilk and in the maternal diet with IgG4 levels in infants which indicate development of immune-tolerance further support this. ${ }^{28}$ Another important risk is low intake of protein, carbohydrate, vitamins and especially calcium which may result in stunting and growth failure. ${ }^{29,30}$

Despite the observed theoretical and practical weaknesses, physicians were adequately knowledgeable in many areas. First of all, the categories of food products that are most commonly restricted; i.e. dairy, peanut, tree nuts, egg, seafood and legumes, fruits are most common allergens that have been shown to cause symptoms in Turkish children. ${ }^{31}$ Secondly, the practice of restriction was observed to be more liberal in the categories of dairy and tree nuts. Only a fraction of those restricting $\mathrm{CM}$ also restrict the products that contain similar proteins as yoghurt, cheese, butter and the baked forms. Similarly, peanut is restricted more commonly than other tree nuts (Fig. 5). Such an approach is favorable since, of the patients with cow's milk protein allergy (CMPA), $70-80 \%$ tolerate baked milk products and $50 \%$ tolerate cheese, yoghurt and butter. ${ }^{18}$ It is argued that inclusion of these products also facilitate the development of tolerance, and improve anxiety and social withdrawal.,18

In addition, majority of the physicians were aware of the common symptoms of both $\operatorname{IgE}$ and non-IgE mediated FA. Most pediatricians prescribed calcium supplementation to mothers with CM restrictions in accordance with relevant guidelines. ${ }^{4,32}$ Finally, the practice of weaning was noted to be appropriate with only $10 \%$ of physicians delaying complementary feeding beyond 6 months. ${ }^{31}$ However, although 
most physicians introduced complementary foods by 6 months, they regarded themselves as delaying introduction in a separate question. A confusion regarding the definition of delayed introduction was noted herein which may mean an introduction of a smaller number of foods at 6 months or later.

The limitations of this study include the small sample size of this study that can hardly reflect all practices in the country. Most participants practiced in bigger cities of Turkey and their resources may be different from the clinical settings in rural areas. Most of the pediatricians were younger than 50 years old. While this suggests our sample had less clinical experience, the impacts of current residency training were reflected more effectively. In addition, our analysis included fewer pediatric allergists and gastroenterologists than general pediatricians. Analyzing them as a single group could have resulted in higher knowledge levels.

In conclusion, FA practices had both strengths and weaknesses. Our results demonstrate a significant concern towards FA, which was reflected in clinical practice as excessive and extensive diet restrictions which were not guided by clinical information and confirmation. The two most important endpoints that should be raised to the physicians are the importance of using appropriate diagnostic methods as well as the recent advances in treatment and prevention of FA.

\section{Supplementary data is available at:}

http://www.turkishjournalpediatrics.org/ uploads/turkjped.2021.01.013.S1.pdf

\section{REFERENCES}

1. Prescott SL, Pawankar R, Allen KJ, et al. A global survey of changing patterns of food allergy burden in children. World Allergy Organ J 2013; 6: 21.

2. Gupta RS, Springston EE, Warrier MR, et al. The prevalence, severity, and distribution of childhood food allergy in the United States. Pediatrics 2011; 128: e9-e17.
3. Jackson KD, Howie LD, Akinbami LJ. Trends in allergic conditions among children: United States, 1997-2011. NCHS Data Brief 2013; 121: 1-8.

4. Boyce JA, Assa'ad A, Burks AW, et al; NIAIDSponsored Expert Panel. Guidelines for the diagnosis and management of food allergy in the United States: report of the NIAID-sponsored expert panel. J Allergy Clin Immunol 2010; 126(Suppl 6): S1-S58.

5. Mazzocchi A, Venter C, Maslin K, Agostoni C. The role of nutritional aspects in food allergy: prevention and management. Nutrients 2017; 9: 850.

6. Du Toit G, Roberts G, Sayre PH, et al; LEAP Study Team Randomized trial of peanut consumption in infants at risk for peanut allergy. N Engl J Med 2015; 372: 803-813.

7. Natsume O, Kabashima S, Nakazato J, et al; PETIT Study Team. Two-step egg introduction for prevention of egg allergy in high-risk infants with eczema (PETIT): a randomised, double-blind, placebo-controlled trial. Lancet 2017; 389: 276-286.

8. Meyer R, De Koker C, Dziubak R, et al. Malnutrition in children with food allergies in the UK. J Hum Nutr Diet 2014; 27: 227-235.

9. Christie L, Hine RJ, Parker JG, Burks W. Food allergies in children affect nutrient intake and growth. J Am Diet Assoc 2002; 102: 1648-1651.

10. Arik Yilmaz E, Cavkaytar O, Buyuktiryaki B, et al. Factors affecting food allergy-related quality of life from parents' perception in Turkish children. Allergy Asthma Immunol Res 2018; 10: 379-386.

11. Cummings AJ, Knibb RC, King RM, Lucas JS. The psychosocial impact of food allergy and food hypersensitivity in children, adolescents and their families: a review. Allergy 2010; 65: 933-945.

12. Yuce A, Dalgic B, Cullu-Cokugras F, et al. Cow's milk protein allergy awareness and practice among Turkish pediatricians: a questionnaire-survey. Turk J Pediatr 2017; 59: 233-243.

13. Gupta RS, Springston EE, Kim JS, et al. Food allergy knowledge, attitudes, and beliefs of primary care physicians. Pediatrics 2010; 125: 126-132.

14. Morawetz DY, Hiscock H, Allen KJ, Davies S, Danchin MH. Management of food allergy: a survey of Australian paediatricians. J Paediatr Child Health 2014; 50: 432-437.

15. Baricic TV, Catipovic M, Cetinic EL, Krmek V, Horvat I. Parental perception, prevalence and primary care physicians' knowledge on childhood food allergy in Croatia. Children (Basel) 2015; 2: 305-316. 
16. Al-Herz W, Husain K, Al-Khabaz A, Moussa MA, Al-Refaee F. Awareness of food allergies: a survey of pediatricians in Kuwait. BMC Pediatr 2017; 17: 11.

17. Kaila M, Vanto T, Valovirta E, Koivikko A, JuntunenBackman K. Diagnosis of food allergy in Finland: survey of pediatric practices. Pediatr Allergy Immunol 2000; 11: 246-249.

18. Sackesen C, Suárez-Fariñas M, Silva R, et al. A new luminex-based peptide assay to identify reactivity to baked, fermented, and whole milk. Allergy 2019; 74: 327-336.

19. Tan-Lim CSC, Esteban-Ipac NAR. Probiotics as treatment for food allergies among pediatric patients: a meta-analysis. World Allergy Organ J 2018; 11: 25 .

20. Meglio P, Giampietro PG, Carello R, Gabriele I, Avitabile S, Galli E. Oral food desensitization in children with IgE-mediated hen's egg allergy: a new protocol with raw hen's egg. Pediatr Allergy Immunol 2013; 24: 75-83.

21. Yrjänä JMS, Bloigu R, Kulmala P. Parental confusion may result when primary health care professionals show heterogeneity in their knowledge, attitudes, and perceptions regarding infant nutrition, food allergy, and atopic dermatitis. Allergol Immunopathol (Madr) 2018; 46: 326-333.

22. Muraro A, Werfel T, Hoffmann-Sommergruber K, et al; EAACI Food Allergy and Anaphylaxis Guidelines Group. EAACI food allergy and anaphylaxis guidelines: diagnosis and management of food allergy. Allergy 2014; 69: 1008-1025.

23. Koletzko S, Niggemann B, Arato A, et al; European Society of Pediatric Gastroenterology, Hepatology, and Nutrition. Diagnostic approach and management of cow's-milk protein allergy in infants and children: ESPGHAN GI Committee practical guidelines. J Pediatr Gastroenterol Nutr 2012; 55: 221-229.
24. Australasian Society of Clinical Immunology and Allergy. Nutritional Management of Food Allergy. https://www.allergy.org.au/images/ stories/pospapers/ASCIA_HPIP_food_allergy_ September_2013.pdf. (Accessed July 4, 2018).

25. Lack G. Epidemiologic risks for food allergy. J Allergy Clin Immunol 2008; 121: 1331-1336.

26. Perkin MR, Logan K, Marrs T, et al; EAT Study Team. Enquiring about tolerance (EAT) study: feasibility of an early allergenic food introduction regimen. J Allergy Clin Immunol 2016; 137: 1477-1486.e8.

27. Katz Y, Rajuan N, Goldberg MR, et al. Early exposure to cow's milk protein is protective against IgE-mediated cow's milk protein allergy. J Allergy Clin Immunol 2010; 126: 77-82.e1.

28. Metcalfe JR, Marsh JA, D'Vaz N, et al. Effects of maternal dietary egg intake during early lactation on human milk ovalbumin concentration: a randomized controlled trial. Clin Exp Allergy 2016; 46: 1605-1613.

29. Sinai T, Goldberg MR, Nachshon L, et al. Reduced final height and inadequate nutritional intake in cow's milk allergic young adults. J Allergy Clin Immunol Pract 2019; 7: 509-515.

30. Meyer R, Wright K, Vieira MC, et al. International survey on growth indices and impacting factors in children with food allergies. J Hum Nutr Diet 2019; 32: $175-184$

31. Greer FR, Sicherer SH, Burks AW, American Academy of Pediatrics Committee on Nutrition; American Academy of Pediatrics Section on Allergy and Immunology.Effects of early nutritional interventions on the development of atopic disease in infants and children: the role of maternal dietary restriction, breastfeeding, timing of introduction of complementary foods, and hydrolyzed formulas. Pediatrics 2008; 121: 183-191.

32. Fiocchi A, Dahda L, Dupont C, Campoy C, Fierro V, Nieto A. Cow's milk allergy: towards an update of DRACMA guidelines. World Allergy Organ J 2016; 9: 35 . 